POLONICI MATHEMATICI

$99.1(2010)$

\title{
Existence and multiplicity results for a nonlinear stationary Schrödinger equation
}

\author{
by Danila Sandra Moschetto (Catania)
}

\begin{abstract}
We revisit Kristály's result on the existence of weak solutions of the Schrödinger equation of the form

$$
-\Delta u+a(x) u=\lambda b(x) f(u), \quad x \in \mathbb{R}^{N}, u \in H^{1}\left(\mathbb{R}^{N}\right),
$$

where $\lambda$ is a positive parameter, $a$ and $b$ are positive functions, while $f: \mathbb{R} \rightarrow \mathbb{R}$ is sublinear at infinity and superlinear at the origin. In particular, by using Ricceri's recent three critical points theorem, we show that, under the same hypotheses, a much more precise conclusion can be obtained.
\end{abstract}

1. Introduction and statement of the main result. Sufficient conditions which ensure the multiplicity of weak solutions for nonlinear stationary Schrödinger-like equations have recently been proposed in the literature. In particular, Kristály [K] considers the Schrödinger equation of the form $\left(P_{\lambda}\right) \quad-\Delta u+a(x) u=\lambda b(x) f(u), \quad x \in \mathbb{R}^{N}, u \in H^{1}\left(\mathbb{R}^{N}\right)$,

with a positive parameter $\lambda$. He assumes that the potentials $a$ and $b$ satisfy the following conditions:

( $\widetilde{a}) a \in L_{\mathrm{loc}}^{\infty}\left(\mathbb{R}^{N}\right), \operatorname{ess}_{\inf _{\mathbb{R}^{N}}} a>0$ and for any $M>0$ and any $r>0$,

$$
\operatorname{mes}\left(\left\{x \in B_{r}(y): a(x) \leq M\right\}\right) \rightarrow 0 \quad \text { as }|y| \rightarrow+\infty
$$

where "mes" stands for the Lebesgue measure and $B_{r}(y)$ denotes the open ball in $\mathbb{R}^{N}$ with center $y$ and radius $r>0$.

$(\widetilde{b}) b \in L^{1}\left(\mathbb{R}^{N}\right) \cap L^{\infty}\left(\mathbb{R}^{N}\right), b \geq 0$ and

$$
\sup _{R>0} \underset{|x| \leq R}{\operatorname{essinf}} b(x)>0 .
$$

$\left(\widetilde{f}_{0}\right) f \in C^{0}(\mathbb{R})$ and there exist $\mathcal{C}>0$ and $\left.q \in\right] 0,1[$ such that

$$
|f(s)| \leq \mathcal{C}|s|^{q} \quad \text { for each } s \in \mathbb{R} .
$$

2010 Mathematics Subject Classification: Primary 35J61.

Key words and phrases: nonlinear Schrödinger equations, multiple solutions. 
$\left(\widetilde{f}_{1}\right) f(s)=o(|s|)$ as $s \rightarrow 0$.

$\left(\widetilde{f}_{2}\right) \sup _{s \in \mathbb{R}} F(s)>0$ where $F(s)=\int_{0}^{s} f(t) d t$.

Following the suggestions of Bartsch and Wang ([BW]), due to $(\widetilde{a})$, Kristály defines the Hilbert space

$$
E=\left\{u \in H^{1}\left(\mathbb{R}^{N}\right): \int_{\mathbb{R}^{N}} a(x) u^{2} d x<+\infty\right\}
$$

endowed with the inner product

$$
(u, v)_{E}=\int_{\mathbb{R}^{N}}(\nabla u \cdot \nabla v+a(x) u v) d x \quad \text { for } u, v, \in E
$$

and consequently with the induced norm which we denote by $\|\cdot\|$. The condition $(\widetilde{a})$ implies that the space $E$ can be continuously embedded into $L^{\ell}\left(\mathbb{R}^{N}\right)$ whenever $2 \leq \ell \leq 2^{*}$ and the embedding is compact when $2 \leq$ $\ell<2^{*}$ (see [Ba]). Here, $2^{*}$ denotes the critical Sobolev exponent, i.e., $2^{*}=$ $2 N /(N-2)$ for $N \geq 3$ and $2^{*}=+\infty$ for $N=1,2$. By applying a result established by Bonanno [B], Kristály has proved in $[\mathrm{K}]$ that $\left(P_{\lambda}\right)$ admits at least two solutions in $E$, provided that $\lambda$ belongs to a suitable open interval. The aim of the present paper is to significantly improve Kristály's result, showing that, essentially under the same hypotheses, a more exact conclusion can be reached. Denoting by $\mathcal{A}$ the class of all Carathéodory functions $g: \mathbb{R}^{N} \times \mathbb{R} \rightarrow \mathbb{R}$ such that the functional

$$
\mathcal{G}(u)=\int_{\mathbb{R}^{N}}\left(\int_{0}^{u(x)} g(x, t) d t\right) d x
$$

belongs to $C^{1}(E)$ and has compact derivative, our main result reads as follows:

Theorem 1.1. Assume $(\widetilde{a}),(\widetilde{b}),\left(\widetilde{f}_{0}\right),\left(\widetilde{f}_{1}\right)$, and $\left(\widetilde{f}_{2}\right)$. Then, setting

$$
\gamma=\frac{1}{2} \inf \left\{\frac{\|u\|^{2}}{\int_{\mathbb{R}^{N}} b(x) F(u(x)) d x}: u \in E, \int_{\mathbb{R}^{N}} b(x) F(u(x)) d x>0\right\},
$$

for each compact interval $[c, d] \subset] \gamma,+\infty[$ there exists a number $r>0$ with the following property: for every $\lambda \in[c, d]$ and every $g \in \mathcal{A}$ there exists $\delta>0$ such that, for each $\mu \in[0, \delta]$, the problem

$$
\left(P_{\lambda, \mu}\right) \quad-\Delta u+a(x) u=\lambda b(x) f(u)+\mu g(x, u), \quad x \in \mathbb{R}^{N}, u \in H^{1}\left(\mathbb{R}^{N}\right),
$$

has at least three weak solutions whose norms in $E$ are less than $r$.

REMARK. This result covers, as a particular case, the problem studied by Kristály $[\mathrm{K}]$. Here, we prove it by a different method and we provide further information both on the size and location of the set containing the 
parameter $\lambda$ and the location of the possible weak solutions of the problem at issue.

2. Proof of Theorem 1.1. First, we recall a theorem from $[\mathrm{R}]$ which is the basic tool in the proof of our result. In the following, if $X$ is a real Banach space, the symbol $\mathcal{W}_{X}$ denotes the class of all functionals $I: X \rightarrow \mathbb{R}$ having the following property: if $\left\{u_{n}\right\}$ is a sequence in $X$ converging weakly to $u \in X$ and $\liminf _{n \rightarrow+\infty} I\left(u_{n}\right) \leq I(u)$, then $\left\{u_{n}\right\}$ has a subsequence converging strongly to $u$.

TheOREM 2.1 ([, Theorem 2]). Let $X$ be a separable and reflexive real Banach space; $\Phi: X \rightarrow \mathbb{R}$ a coercive, sequentially weakly lower semicontinuous $C^{1}$ functional, belonging to $\mathcal{W}_{X}$, bounded on each bounded subset of $X$ and whose derivative admits a continuous inverse on $X^{*}$; and $J: X \rightarrow \mathbb{R}$ a $C^{1}$ functional with compact derivative. Assume that $\Phi$ has a strict local minimum at $x_{0}$ with $\Phi\left(x_{0}\right)=J\left(x_{0}\right)=0$. Finally, setting

$$
\begin{aligned}
& \alpha=\max \left\{0, \limsup _{\|x\| \rightarrow+\infty} \frac{J(x)}{\Phi(x)}, \limsup _{x \rightarrow x_{0}} \frac{J(x)}{\Phi(x)}\right\}, \\
& \beta=\sup _{x \in \Phi^{-1}(] 0,+\infty[)} \frac{J(x)}{\Phi(x)}
\end{aligned}
$$

assume that $\alpha<\beta$. Then, for each compact interval $[c, d] \subset] 1 / \beta, 1 / \alpha[$ (with the conventions $\frac{1}{0}=+\infty, \frac{1}{+\infty}=0$ ) there exists $r>0$ with the following property: for every $\lambda \in[c, d]$ and every $C^{1}$ functional $\Psi: X \rightarrow \mathbb{R}$ with compact derivative, there exists $\delta>0$ such that, for each $\mu \in[0, \delta]$, the equation

$$
\Phi^{\prime}(x)=\lambda J^{\prime}(x)+\mu \Psi^{\prime}(x)
$$

has at least three solutions whose norms are less than $r$.

To use this theorem in our particular case, we begin by defining the functional $\mathcal{F}: E \rightarrow \mathbb{R}$ as

$$
\mathcal{F}(u)=\int_{\mathbb{R}^{N}} b(x) F(u(x)) d x
$$

for each $u \in E$. Standard arguments based on the hypothesis $(\widetilde{a})$ and on the fact that $E$ is continuously embedded in $L^{\ell}\left(\mathbb{R}^{N}\right)$ when $2 \leq \ell \leq 2^{*}$ show that the functional $\mathcal{F}$ is well defined, it is of class $C^{1}$, and satisfies

$$
\mathcal{F}^{\prime}(u)(v)=\int_{\mathbb{R}^{N}} b(x) f(u(x)) v(x) d x \quad \text { for all } u, v \in E .
$$

Moreover, since the embedding $E \hookrightarrow L^{\ell}\left(\mathbb{R}^{N}\right)$ is compact for $2 \leq \ell<2^{*}, \mathcal{F}^{\prime}$ is a compact operator. In the following, we denote by $\kappa_{\ell}>0$ the Sobolev embedding constant for $E \hookrightarrow L^{\ell}\left(\mathbb{R}^{N}\right)$ where $\ell \in\left[2,2^{*}\right]$. Finally, for any 
$\lambda>0$ and $\mu \geq 0$ we define the functional $\mathcal{H}: E \rightarrow \mathbb{R}$ by

$$
\mathcal{H}(u)=\frac{1}{2}\|u\|^{2}-\lambda \mathcal{F}(u)-\mu \mathcal{G}(u) \quad \text { for all } u \in E .
$$

Obviously, the weak solutions of the problem $\left(P_{\lambda, \mu}\right)$ are the critical points of $\mathcal{H}$.

We are now in a position to prove Theorem 1.1.

Proof of Theorem 1.1. We apply Theorem 2.1 for $X=E, \Phi(u)=\frac{1}{2}\|u\|^{2}$ and $J=\mathcal{F}$. Note that $\Phi$ is a coercive, sequentially weakly lower semicontinuous $C^{1}$ functional which belongs to $\mathcal{W}_{E}$. The latter assertion is a classical result, since the space $E$ is uniformly convex and $\Phi(u)=h(\|u\|)$ with $h(t)=\frac{1}{2} t^{2}:[0,+\infty[\rightarrow \mathbb{R}$, which is a continuous and strictly increasing function. Because $\Phi$ is continuous, it is bounded on each bounded subset of $E$, its derivative is a homeomorphism between $E$ and its dual (see [Z, Theorem 26. A]), and the hypotheses on $J$ of Theorem 2.1 are satisfied as well. Putting $u_{0}=\theta_{E}$, where $\theta_{E}$ is the zero element of $E$, observe that $\Phi$ has at $u_{0}$ the only global minimum. Moreover, if $u \neq \theta_{E}$ then $\Phi(u)>0$ by $(\widetilde{a})$ and $\Phi\left(u_{0}\right)=J\left(u_{0}\right)=0$. Now, we fix a number $\epsilon>0$; in view of $\left(\widetilde{f}_{0}\right)$ and $\left(\widetilde{f}_{1}\right)$ there exist $\rho_{1}, \rho_{2}$ with $0<\rho_{1}<\rho_{2}$ such that

$$
b(x) F(s)<\epsilon a(x)|s|^{2}
$$

for a.e. $x \in \mathbb{R}^{N}$ and all $s \in \mathbb{R} \backslash\left(\left[-\rho_{2},-\rho_{1}\right] \cup\left[\rho_{1}, \rho_{2}\right]\right)$. Then, as $F$ is bounded on $\left[-\rho_{2},-\rho_{1}\right] \cup\left[\rho_{1}, \rho_{2}\right]$, we can choose $\mathcal{D}>0$ and $2<q<2^{*}$ in such a way that

$$
b(x) F(s)<\epsilon a(x)|s|^{2}+\mathcal{D}|s|^{q}
$$

for a.e. $x \in \mathbb{R}^{N}$ and all $s \in \mathbb{R}$. Thus, by continuous embedding,

$$
\mathcal{F}(u) \leq \epsilon\|u\|^{2}+\mathcal{D} \kappa_{q}^{q}\|u\|^{q}
$$

for all $u \in E$. Hence,

$$
\limsup _{u \rightarrow 0} \frac{2 \mathcal{F}(u)}{\|u\|^{2}} \leq 2 \epsilon
$$

Further, by (2.1) again, for each $u \in E \backslash\left\{\theta_{E}\right\}$, we obtain

$$
\begin{aligned}
\frac{\mathcal{F}(u)}{\|u\|^{2}} & =\frac{\int_{\left(|u| \leq \rho_{2}\right)} b(x) F(u(x)) d x}{\|u\|^{2}}+\frac{\int_{\left(|u|>\rho_{2}\right)} b(x) F(u(x)) d x}{\|u\|^{2}} \\
& \leq \frac{\sup _{\left[-\rho_{2}, \rho_{2}\right]} F \int_{\mathbb{R}^{N}} b(x) d x}{\|u\|^{2}}+\epsilon .
\end{aligned}
$$

So, we get

$$
\limsup _{\|u\| \rightarrow+\infty} \frac{2 \mathcal{F}(u)}{\|u\|^{2}} \leq 2 \epsilon
$$


Since $\epsilon$ is arbitrary, from $(2.2)$ and $(2.3)$ it follows that

$$
\max \left\{\limsup _{\|u\| \rightarrow+\infty} \frac{2 \mathcal{F}(u)}{\|u\|^{2}}, \limsup _{u \rightarrow 0} \frac{2 \mathcal{F}(u)}{\|u\|^{2}}\right\} \leq 0 .
$$

Thus, by using the notation of Theorem 2.1, we have $\alpha=0$ and by our assumption $0<\beta \leq+\infty$. Therefore, for $\gamma=1 / \beta$, the conclusion follows from Theorem 2.1 with $\Psi=\mathcal{G}$.

EXAMPLE 2.2. Let $\kappa, h$ and $\xi$ be arbitrary real positive. We choose $f: \mathbb{R} \rightarrow \mathbb{R}$ defined by

$$
f(s)= \begin{cases}s|s|[4 m|s|+3 n], & |s| \leq \xi, \\ \kappa s e^{-h|s|}, & |s| \geq \xi\end{cases}
$$

where

$$
m=m(\kappa, h, \xi)=-\frac{\kappa e^{-h \xi}(h \xi+1)}{4 \xi^{2}}, \quad n=n(\kappa, h, \xi)=\frac{\kappa e^{-h \xi}(2+h \xi)}{3 \xi} .
$$

Then, we take as potentials $a(x)=|x|^{2}+\ell$ with $\ell$ a positive constant and $b(x)=e^{-|x|^{2}}, x \in \mathbb{R}^{N}$. It follows easily that the assumptions $(\widetilde{a}),(\widetilde{b}),\left(\widetilde{f}_{0}\right)$, $\left(\widetilde{f}_{1}\right)$ and $\left(\widetilde{f}_{2}\right)$ of Theorem 1.1 hold.

\section{References}

[Ba] T. Bartsch, A. Pankov and Z.-Q. Wang, Nonlinear Schrödinger equations with steep potential well, Comm. Contemp. Math. 4 (2001), 549-569.

[BW] T. Bartsch and Z.-Q. Wang, Existence and multiplicity results for some superlinear elliptic problems on $\mathbb{R}^{N}$, Comm. Partial Differential Equations 20 (1995), 17251741 .

[B] G. Bonanno, Some remarks on a three critical points theorem, Nonlinear Anal. 54 (2003), 651-665.

[K] A. Kristály, Multiple solutions of a sublinear Schrödinger equation, Nonlinear Differential Equations Appl. 14 (2007), 291-301.

[R] B. Ricceri, A further three critical points theorem, Nonlinear Anal. 71 (2009), 4151-4157.

[Z] E. Zeidler, Nonlinear Functional Analysis and its Applications, Vol. II/B, Springer, 1985.

Danila Sandra Moschetto

Department of Mathematics and Computer Science

University of Catania

Viale A. Doria, 6

95125 Catania, Italy

E-mail: moschetto@dmi.unict.it 
